\title{
Multiple Eruptive Milia and Milia En Plaque in a 21-Year-Old Man
}

\author{
Ji Yeoun Shin', Sung Yul Lee'2, Young Lip Park', Kyu Uang Whang ${ }^{3}$, Jung Eun Kim² \\ 'Department of Dermatology, Soonchunhyang University Bucheon Hospital, Soonchunhyang University College of Medicine, Bucheon; ${ }^{2}$ Department of \\ Dermatology, Soonchunhyang University Cheonan Hospital, Soonchunhyang University College of Medicine, Cheonan; ${ }^{3}$ Department of Dermatology, \\ Soonchunhyang University Seoul Hospital, Soonchunhyang University College of Medicine, Seoul, Korea
}

\begin{abstract}
Milia are benign primary or secondary keratinous cysts. Multiple eruptive milia (MEM) and milia en plaque (MEP) are rarely reported. A 21-year-old male presented with multiple skin-colored papules on the abdomen and both acral regions, and erythematous plaques featuring similar papules on the wrist and the dorsa of the feet. A biopsy confirmed MEP and he was diagnosed with both MEM and MEP. We thus report a rare case of simultaneous MEM and MEP.
\end{abstract}

Keywords: Keratosis; Milia; Skin diseases

\section{INTRODUCTION}

Milia are benign keratinous cysts that arise spontaneously either primarily or secondarily [1]. Idiopathic multiple eruptive milia (MEM) are rare, characterized by sudden onset on the head, neck, trunk, and hands. Milia en plaque (MEP) is a variant of the condition, characterized by multiple milia on an erythematous background [2]. Both MEM and MEP are rare, with few reported cases. Only two case reports of simultaneous occurrence have been reported in the English-language literature [2,3]. We report a rare case of simultaneous MEM and MEP.

\section{CASE REPORT}

A 21-year-old male presented with tiny, multiple skin-colored papules on the abdomen and both acral regions. The lesions had first appeared 2 years prior and had spread rapidly. He had no history of trauma, drug intake, or skin disease. The family history was non-specific. Physical examination revealed multiple, firm, 1-3-mm-diameter skin-colored papules on the trunk (Fig. 1), hands, and feet (Fig. 2A, B). Also, uniquely, some erythematous plaques containing such papules were found on the wrist (Fig. 3). We performed punch biopsy of the left wrist. Histological exami- nation revealed two small cysts filled with concentric lamellar keratin in the superficial dermis (Fig. 4). Multinucleated giant cells and lymphohistiocytes were evident around the cysts, suggesting rupture. Although cyst-lining cells were absent, the keratinous material and the small well-formed lumina were consistent with milia.

The patient was diagnosed with both MEM and MEP based on the clinical and histological features. He received systemic antibiotics, triggering gradual remission.

\section{DISCUSSION}

Milia are benign keratinous cysts thought to originate in the infundibula of vellus hairs, or sebaceous glands and the eccrine sweat ducts [4]. Milia may be either primary or secondary in nature. Spontaneous primary milia are common on the face and eyelids of newborns. Secondary milia may develop anywhere after trauma, a blistering disease, radiotherapy, or topical therapy with glucocorticoids or 5-fluorouracil [4]. Primary milia are of several forms, including MEM, MEP, and nodular grouped milia [4].

Idiopathic MEM is characterized by an "eruption" onset of multiple milia over weeks-to-months in the absence of any obvious trigger. The lesions are more numerous than those of simple 
benign primary milia [4]. Histological examination of biopsy specimens reveals cysts in the superficial dermis; the cysts are lined by stratified squamous epithelium and contain concentric keratin lamellae [1]. The etiology of MEM is unclear; MEM may lack a specific causative factor. MEM may be inherited, and/or related to the development of genodermatoses such as Bazex, Rombo, or Brooke-Spiegler syndrome; atrichia with papular lesions; or basal cell nevus syndrome [5]. Rare familial autosomal dominant transmission has also been reported [6].

MEP is a rare form of milia first described in 1903 by Balzer and Fouquet [7]; the term "MEP" was first coined by Hubler et al. [8] in 1978. Since that time, fewer than 40 cases of MEP have been reported [9]. MEP is characterized by multiple tiny papules on an erythematous background, mainly in the periauricular or periorbital area [2]. Histologically, MEP features cysts surrounded by

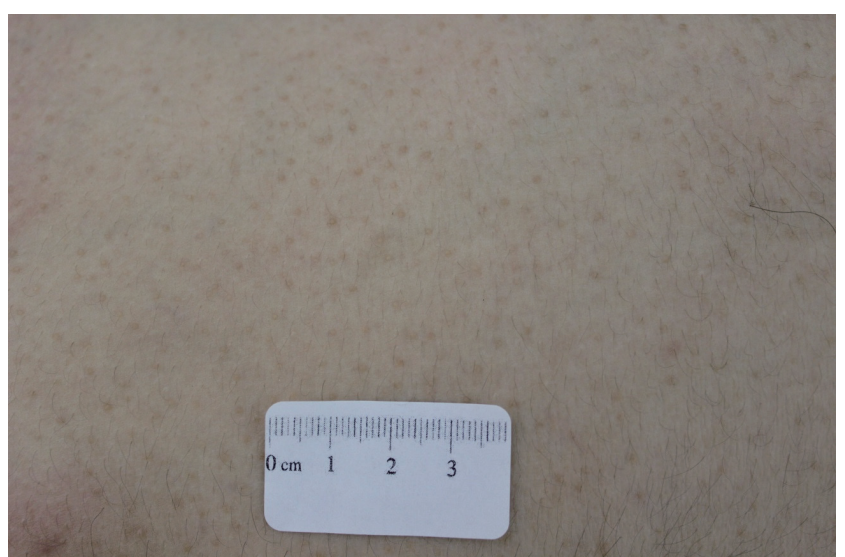

Fig. 1. Multiple, flesh-colored, and tiny papules are scattered on the patient's abdomen.

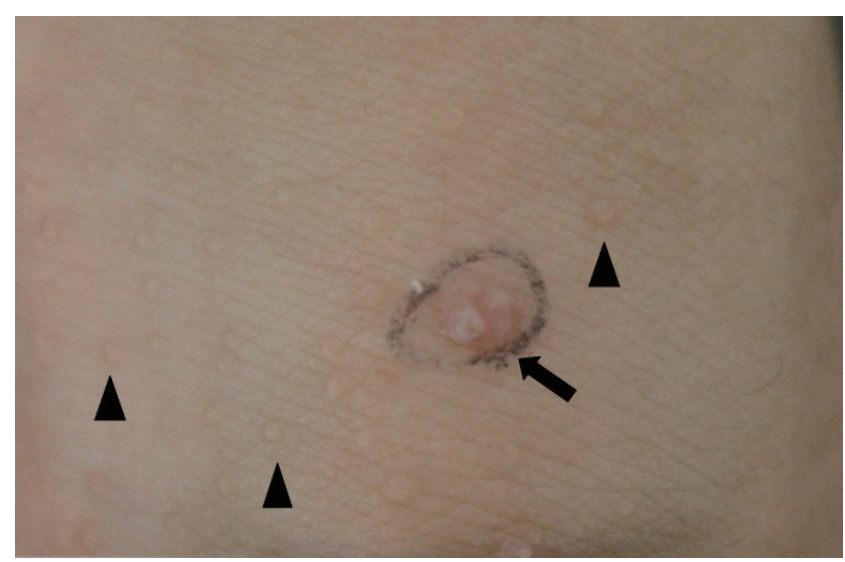

Fig. 3. Arrow indicates grouped papules presenting clinical features of milia en plaque and arrowheads indicate idiopathic multiple milia.

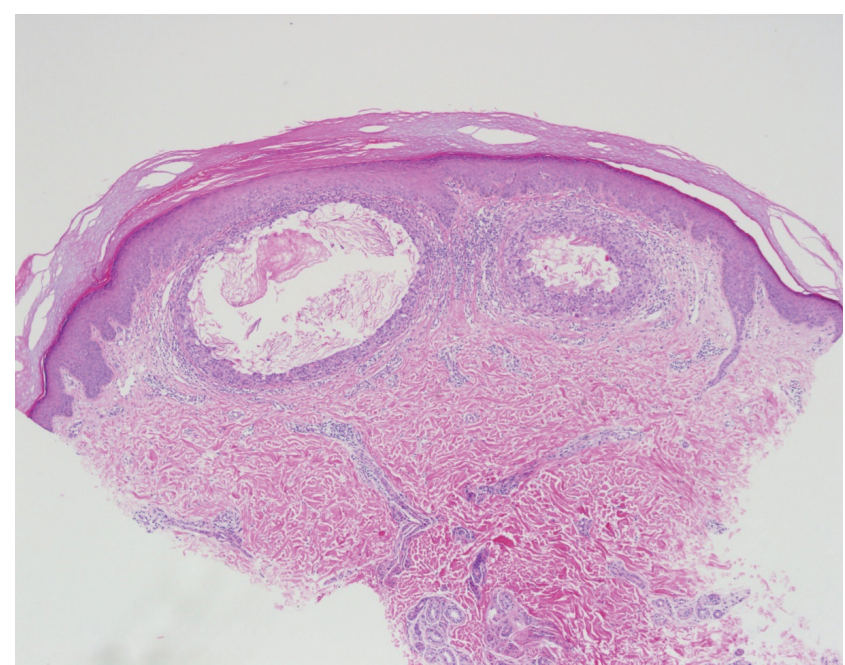

Fig. 4. Two well-formed small cysts with keratinous material were observed in superficial dermis $(\mathrm{H} \& \mathrm{E}, \times 20)$.
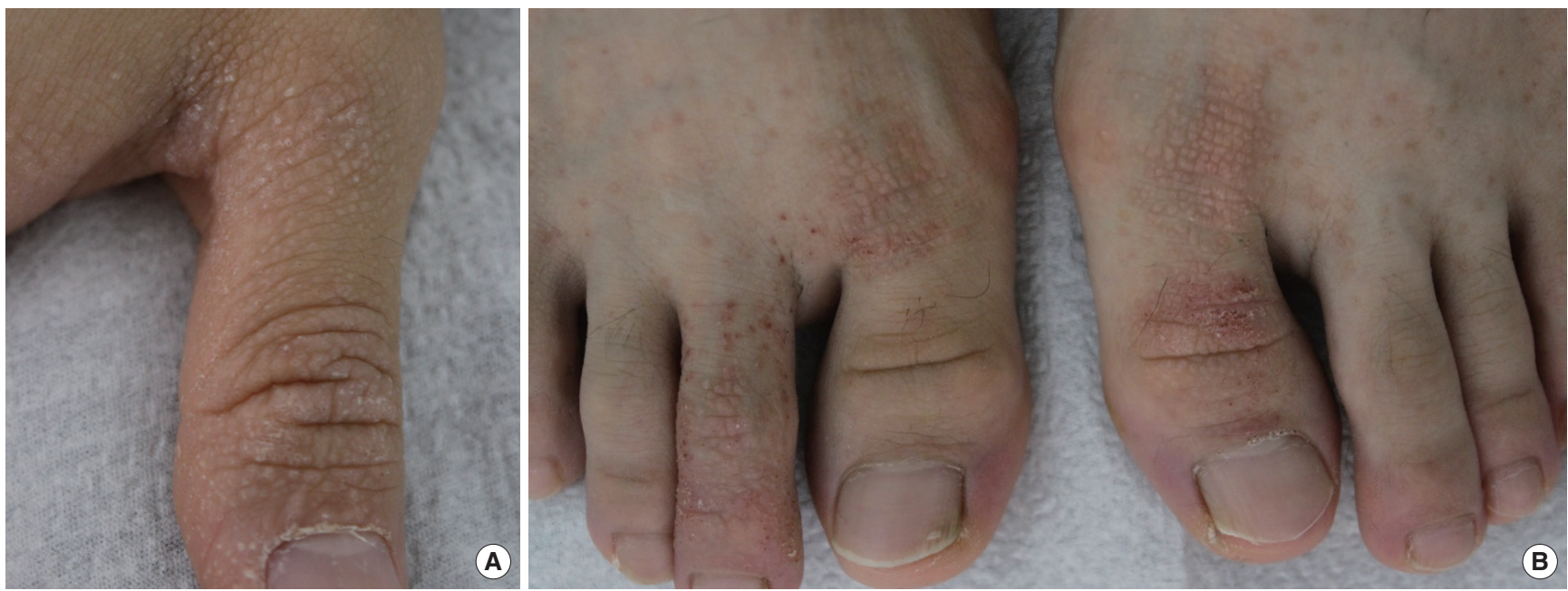

Fig. 2. (A, B) A number of firm and tiny papules, some of them grouped on the erythematous background, are noted on both hands and feet. 
mild-to-dense mononuclear and lymphocytic infiltrates [4]. The etiology and pathogenesis of MEP remain unknown, although MEP may be associated with pseudoxanthoma elasticum, discoid lupus erythematosus, and renal transplantation [10]. Recently, a patient with MEP evident at birth accompanied by a Blaschkoid extension on the parietal scalp has been reported [9]. MEP is thought to spontaneously regress in adults and no recognized treatment has been described. However, in practice, topical retinoids and steroids, oral antibiotics, and $\mathrm{CO}_{2}$ and erbium-yttriumaluminum-garnet (Er:YAG) lasers have been used to treat MEP; the success rates varied among small numbers of patients [4].

Although spontaneous milia regression has been reported, and although milia are benign and asymptomatic, cosmetic concerns are in play. Simple evacuation via incision and curettage effectively treat limited numbers of milia. However, no gold standard treatment for multiple milia has yet been established; the few relevant reports feature excision, curettage, cryotherapy, oral minocycline or azithromycin, topical retinoin, and carbon dioxide and Er:YAG laser treatments [3]. The outcomes were similar.

To the best of our knowledge, simultaneous MEM and MEP has been described by Jung et al. [2] and Jung et al. [3]. In these cases, the lesional magnitude and sporadic spread were characteristic of MEM, but the morphology and histopathological data were indic- ative of MEP. Our present case exhibited the clinical features of both MEM and MEP. In summary, we report a patient with a 2-year history of abdominal MEM and MEP on both extremities, with no definite causative factor.

\section{REFERENCES}

1. Fett N, Nevas J. Multiple eruptive milia. Cutis 2013;91:191-2.

2. Jung SW, Park IH, Lee W, Seol JE, Kim H, Sung HS. Multiple eruptive milia and milia en plaque on the hands and feet of a 2-month-old female. J Dermatol 2015;42:931-2.

3. Jung YS, Cho SH, Lee JD, Kim HS. Multiple eruptive milia coexisting with milia en plaque. J Clin Investig Dermatol 2016;4:1.

4. Berk DR, Bayliss SJ. Milia: a review and classification. J Am Acad Dermatol 2008;59:1050-63.

5. Cho E, Cho SH, Lee JD. Idiopathic multiple eruptive milia occurred in unusual sites. Ann Dermatol 2010;22:465-7.

6. Langley RG, Walsh NM, Ross JB. Multiple eruptive milia: report of a case, review of the literature, and a classification. J Am Acad Dermatol 1997;37(2 Pt 2):353-6.

7. Balzer F, Fouquet C. Milium confluent retroauriculaire bilateral. Bull Soc Fr Dermatol Syphiligr 1903;14:361-2.

8. Hubler WR Jr, Rudolph AH, Kelleher RM. Milia en plaque. Cutis 1978; 22:67-70.

9. Ghosh S, Sangal S. Congenital milia en plaque on scalp. Indian J Dermatol 2015;60:105.

10. Munoz-Martinez R, Santamarina-Albertos A, Sanz-Munoz C, MirandaRomero A. Milia en plaque. Actas Dermosifiliogr 2013;104:638-40. 